\title{
HIF2 $\alpha$ cooperates with RAS to promote lung tumorigenesis in mice
}

\author{
William Y. Kim, ${ }^{1}$ Samanthi Perera, ${ }^{2,3}$ Bing Zhou, ${ }^{1}$ Julian Carretero,,2,3 Jen Jen Yeh,1,4
} Samuel A. Heathcote, ${ }^{1}$ Autumn L. Jackson, ${ }^{1}$ Petros Nikolinakos, ${ }^{5}$ Beatriz Ospina, ${ }^{6}$ George Naumov, ${ }^{7}$ Kathleyn A. Brandstetter, ${ }^{2,3}$ Victor J. Weigman, ${ }^{1}$ Sara Zaghlul, ${ }^{2,3}$ D. Neil Hayes, ${ }^{1}$ Robert F. Padera, ${ }^{8}$ John V. Heymach, ${ }^{5}$ Andrew L. Kung, ${ }^{6}$ Norman E. Sharpless, ${ }^{1,9}$ William G. Kaelin Jr., 2,10 and Kwok-Kin Wong ${ }^{2,3}$

'Lineberger Comprehensive Cancer Center, University of North Carolina, Chapel Hill, North Carolina, USA.

2Department of Medical Oncology, Dana-Farber Cancer Institute, Boston, Massachusetts, USA.

${ }^{3}$ Ludwig Center at Dana-Farber/Harvard Cancer Center, Boston, Massachusetts, USA. ${ }^{4}$ Department of Surgery,

University of North Carolina, Chapel Hill, North Carolina, USA. ${ }^{5}$ Department of Thoracic/Head and Neck Medical Oncology, University of Texas MD Anderson Cancer Center, Houston, Texas, USA. 'Department of Pediatric Oncology, Dana-Farber Cancer Institute and Children's Hospital Boston, Boston, Massachusetts, USA. 'Department of Surgery, Children's Hospital Boston, Boston, Massachusetts, USA. ${ }^{8}$ Department of Pathology, Brigham and Women's Hospital, Boston, Massachusetts, USA. ${ }^{9}$ Department of Genetics, University of North Carolina, Chapel Hill, North Carolina, USA.

${ }^{10}$ Howard Hughes Medical Institute, Boston, Massachusetts, USA.

\begin{abstract}
Members of the hypoxia-inducible factor (HIF) family of transcription factors regulate the cellular response to hypoxia. In non-small cell lung cancer (NSCLC), high HIF2 $\alpha$ levels correlate with decreased overall survival, and inhibition of either the protein encoded by the canonical HIF target gene VEGF or VEGFR2 improves clinical outcomes. However, whether HIF2 $\alpha$ is causal in imparting this poor prognosis is unknown. Here, we generated mice that conditionally express both a nondegradable variant of HIF2 $\alpha$ and a mutant form of $\operatorname{Kras}\left(\operatorname{Kras}^{G 12 D}\right)$ that induces lung tumors. Mice expressing both Hif2a and $\mathrm{Kras}^{\mathrm{G} 12 D}$ in the lungs developed larger tumors and had an increased tumor burden and decreased survival compared with mice expressing only Kras $^{\text {G12D }}$. Additionally, tumors expressing both $\mathrm{Kras}^{\mathrm{G} 12 \mathrm{D}}$ and Hif2a were more invasive, demonstrated features of epithelial-mesenchymal transition (EMT), and exhibited increased angiogenesis associated with mobilization of circulating endothelial progenitor cells. These results implicate HIF2 $\alpha$ causally in the pathogenesis of lung cancer in mice, demonstrate in vivo that HIF $2 \alpha$ can promote expression of markers of EMT, and define HIF $2 \alpha$ as a promoter of tumor growth and progression in a solid tumor other than renal cell carcinoma. They further suggest a possible causal relationship between HIF2 $\alpha$ and prognosis in patients with NSCLC.
\end{abstract}

\section{Introduction}

HIF is a heterodimeric transcription factor composed of a labile $\alpha$ subunit (HIF $\alpha$ ) and a stable $\beta$ subunit (HIF $\beta)(1)$. The human genome encodes 3 HIF $\alpha$ subunit genes (HIF1A, HIF2A, HIF3A) and 3 HIFB genes (1). Under normal oxygen conditions, HIF $\alpha$ subunits bind with the VHL gene product, pVHL, which acts as the substrate recognition component of an E3 ubiquitin ligase complex. pVHL, along with other members of the pVHL ubiquitin ligase complex, polyubiquitinates and destroys HIF $\alpha$ subunits in a proteasome-dependent manner. The binding of HIF $\alpha$ by pVHL is dependent upon the oxygen-dependent, posttranslational hydroxylation of HIF subunits on either of two conserved prolyl residues (1). This prolyl hydroxylation is catalyzed by the oxygen-dependent EGLN (also known as PHD) family of prolyl hydroxylases (2). Thus, HIF $\alpha$ subunits are normally degraded by $\mathrm{pVHL}$ in the presence of oxygen but stabilized under conditions of hypoxia or in the setting of PVHL inactivation. HIF $\alpha$ subunits have a very short half-life (measured in min-

Authorship note: William Y. Kim and Samanthi Perera contributed equally to this work.

Conflict of interest: The authors have declared that no conflict of interest exists. Nonstandard abbreviations used: Ad-Cre, adenovirus encoding Cre recombinase; CEC, circulating endothelial cell; CEP, circulating endothelial progenitor cell; EMT, epithelial-mesenchymal transition; FDR, false discovery rate; GSEA, gene set enrichment analysis; MVD, microvessel density; NSCLC, non-small cell lung cancer. Citation for this article: J. Clin. Invest. 119:2160-2170 (2009). doi:10.1172/JCI38443 utes during normoxia) and therefore are sensitive to changes in protein translation, such as those mediated by the mammalian target of rapamycin (mTOR) (3-5).

While HIF protein stability is modulated primarily by oxygen levels and the pVHL status of cells, HIF mRNA levels and protein synthesis are regulated primarily in an oxygen-independent manner. For example, activation of the PI3K and MAPK pathways via growth factor receptor tyrosine kinases (RTKs), tumor suppressor loss, or oncogene activation have all been shown to result in variable increases in both HIF mRNA and protein levels $(1,6-10)$. HIF accumulation leads to the direct activation of more than 100 target genes (1). While the majority of these genes are involved in the cellular and organismal adaptation to hypoxia, a substantial proportion of these genes are potentially capable of promoting tumor progression. For example, genes involved in angiogenesis (VEGF, PDGF, PIGF), degradation of the extracellular matrix (MMPs), metastasis ( $L O X, C X C R 4, S D F 1)$, epithelial to mesenchymal transition (SNAIL, SIP, ZEB), and immortalization (bTERT) are known HIF target genes $(1,11)$.

Despite sharing similar functional domains and substantial sequence homology, HIF $1 \alpha$ and HIF $2 \alpha$ do not appear to be fully redundant in function. First, while knockout of either Hif1a or Hif2a in mice leads to embryonic lethality, both the timing and cause of death appear to differ. Hifla $a^{-/-}$mice die in mid-gestation and exhibit severe blood vessel defects, whereas $\mathrm{Hif} 2 \mathrm{a}^{-/-}$mice exhibit late embryonic or early postnatal lethality as a consequence of catecholamine 
A

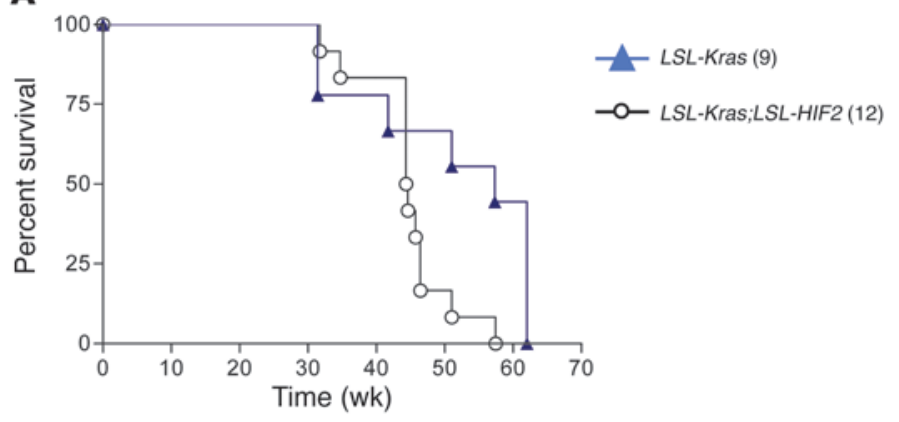

C

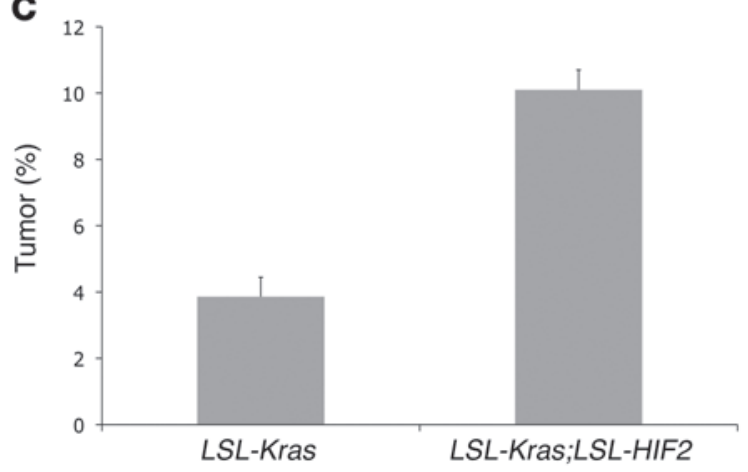

B

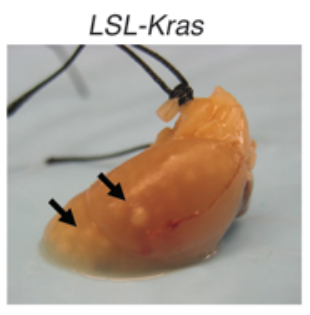

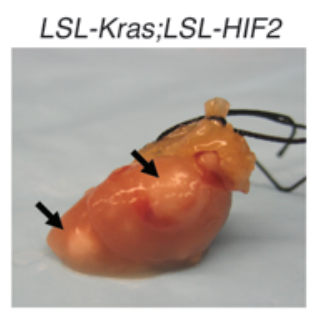

Figure 1

Mice harboring HIF2 $\alpha$-producing lung tumors have a shortened survival and larger tumors. (A) Kaplan-Meier survival curve of cohorts of LSL-Kras $(n=9)$ and LSL-Kras;LSL-HIF2 $(n=12)$ mice $(P=0.036)$. (B) Photomicrographs of representative lung tumors from $L S L-K r a s$ and LSL-Kras;LSL-HIF2 mice. Arrows indicate visible subpleural tumors. (C) Quantification of tumor area as a percentage of lung area. Error bars indicate 1 SEM $(P=0.031)$.

deficiency or lung maturation defects (12-14). Second, the functional differences between HIF $1 \alpha$ and HIF $2 \alpha$ do not appear to be limited to their roles in development, as a recent report also demonstrates that postnatal deletion of Hif1a or Hif2a results in differing phenotypes (15). Finally, studies from our laboratory and others have now demonstrated that HIF $1 \alpha$ and HIF $2 \alpha$ produce overlapping yet distinct gene expression profiles and that certain target genes are uniquely regulated by either HIF1 $\alpha$ or HIF2 $\alpha$ (16-20).

HIF's role in tumorigenesis is best characterized in the context of renal cell carcinoma (RCC), where HIF2 $\alpha$ appears to play a special role (21). $\mathrm{VHL}^{-/-}$renal carcinomas demonstrate obligate expression of HIF $2 \alpha$, but not HIF1 $\alpha$, suggesting a selective pressure to maintain HIF2 $\alpha$ expression $(22,23)$. An apparent switch from HIF $1 \alpha$ to HIF $2 \alpha$ expression occurs in preneoplastic lesions arising in human $V H L^{+/-}$kidneys in association with increasing dysplasia and cellular atypia (24). Finally, in $V H L^{-1-}$ RCCs, HIF $2 \alpha$, rather than HIF1 $\alpha$, appears to be necessary and sufficient for tumor growth (25-28). Thus, at least in VHL-deficient RCC, HIF2 $\alpha$ is the relevant oncogenic HIF family member (29).

Evidence also suggests that hypoxia, HIF, and in particular HIF2 $\alpha$ may play an important role in the pathogenesis and prognosis of lung cancer. Prior work has indicated that intratumoral hypoxia is clearly present, even in some early-stage lung cancers, and correlates with a decreased overall survival $(30,31)$. Furthermore, HIF1A mRNA is one of 3 transcripts that have been validated to stratify early-stage non-small cell lung cancer (NSCLC) patients into groups with different prognoses (32). Interestingly, while multiple studies have shown that HIF $1 \alpha$ is overexpressed at the protein level in a substantial pro- portion (33\%-62\%) of NSCLCs, patients with elevated HIF1 $\alpha$ protein levels do not have a shortened overall survival $(33,34)$.

HIF $2 \alpha$, like HIF $1 \alpha$, is frequently overexpressed at the protein level in NSCLC (50\%) (33). However, overexpression of HIF $2 \alpha$, in contrast to HIF $1 \alpha$, correlates with decreased survival, suggesting that HIF2 $\alpha$ either causes, or is a surrogate marker for, poor clinical outcome (33). Finally, it is notable that bevacizumab, the monoclonal antibody targeting VEGF (a canonical HIF target gene) and sunitinib, the small molecule tyrosine kinase inhibitor that targets VEGFR2 (among other tyrosine kinases), have shown clinical benefit for patients with metastatic NSCLC $(35,36)$.

To evaluate the role of HIF $2 \alpha$ in lung tumorigenesis, we generated cohorts of mice that conditionally expressed mutant Kras $^{G 12 D}$ with or without a conditionally expressed variant of stabilized HIF2 $\alpha$ (HIF2dPA). Our data reveal that lung-specific expression of HIF2dPA on a background of mutant Kras leads to the development of lung cancers with highly pleomorphic histology and features of epithelial-mesenchymal transition (EMT), increased vascularization and blood flow, as well as elevated levels of circulating endothelial cells (CECs) and circulating endothelial progenitor cells (CEPs). Furthermore, mice harboring HIF2dPA-expressing lung cancers exhibited an increased tumor burden, more invasive tumors, and a shortened median survival. These genetic results complement existing human clinical data to implicate HIF $2 \alpha$ as a negative prognostic factor in human NSCLC and demonstrate that HIF $2 \alpha$ can promote tumor growth in tumors other than renal cell carcinoma and specifically in Kras mutant lung carcinoma. 
Table 1

Comparison of lung cancer cohorts

$\begin{array}{lccc} & \text { LSL-KRas } & \text { LSL-KRas; LSL-HIF2 } & \boldsymbol{P} \\ \text { Median survival } & 57 \mathrm{wk} & 42 \mathrm{wk} & 0.03 \\ \text { Mean tumor number (SEM) } & 48(6.5) & 47.3(7.6) & 0.69 \\ \text { Mice with tumor }>3 \mathrm{~mm} & 0 / 17(0 \%) & 10 / 18(55 \%) & <0.001 \\ \text { Mice with local invasion or metastases } & 0 / 40(0 \%) & 2 / 12(16 \%) & 0.05 \\ \text { Tumors with increased stroma } & 1 / 153(0.65 \%) & 25 / 593(4.2 \%) & 0.03 \\ \text { Poorly differentiated tumors } & 4 / 153(2.6 \%) & 45 / 593(7.6 \%) & 0.027\end{array}$

Despite these differences in tumor involvement, there was no significant difference in either proliferation, as measured by $\mathrm{Ki} 67$ staining $(P=0.45$; Supplemental Figure $1 \mathrm{~A}$; supplemental material available online with this article; doi:10.1172/JCI38443DS1), or apoptosis, as measured by TUNEL staining $(P=0.85$; Supplemental Figure 1B), between the tumors of LSL-Kras and LSL-Kras;LSL-HIF2 mice. This may reflect that fact that these measurements were taken at a late time point in the growth of these tumors. Alternatively, it is also possible that the increased

\section{Results}

Expression of HIF2dPA decreases survival of mice with Kras-induced lung tumors. We previously generated knock-in mice that expressed a conditionally activatable form of HA-tagged HIF2 $\alpha$ (HIF2dPA) that escapes recognition by $\mathrm{PVHL}$ by virtue of proline-to-alanine substitutions (hereafter called LSL-HIF2 for simplicity) (16). Specifically, the ROSA26 locus of these mice harbors a Lox-Stop-Lox cassette interposed between the endogenous ROSA26 promoter and the HIF2dPA cDNA, and the levels of HIF2 $\alpha$ expressed are relatively physiologic (16). Therefore, transcription is under control of the ROSA26 promoter but dependent upon Cre-mediated excision of the floxed stop element.

Adenovirus encoding Cre recombinase (Ad-Cre) can be delivered to the murine lung by intranasal administration. Mice harboring a conditionally activatable allele of mutant Kras ${ }^{G 12 D}$, Lox-Stop-Lox Kras $^{G 12 D}$ (hereafter called LSL-Kras) develop multifocal tumors with a relatively long latency and low invasive potential (37-40). To determine the role of HIF $2 \alpha$ in lung tumorigenesis, we intercrossed the LSL-HIF2 mice to LSL-Kras mice to generate cohorts of LSL-Kras;LSL-HIF2 and littermate control LSL-Kras mice. These cohorts were then treated with a low dose of intranasal Ad-Cre $\left(2.5 \times 10^{6} \mathrm{PFU}\right)(38)$. LSL-Kras mice treated with intranasal Ad-Cre developed multiple tumors with a moderate multiplicity and had a median survival of 57 weeks (Figure 1A and Table 1). LSL-Kras;LSL-HIF2 mice also developed lung tumors with approximately the same multiplicity $(P=0.69$; Table 1$)$ but with a shortened median survival of 42 weeks $(P=0.036$; Figure $1 \mathrm{~A}$ and Table $1)$. Therefore, the data indicate that expression of HIF $2 \alpha$ within Kras-induced lung carcinomas does not increase tumor multiplicity but confers a shortened survival.

HIF2 $\alpha$-producing Kras-induced lung tumors are larger and result in an increased lung tumor burden. Gross examination of the visceral pleura of inflated lungs taken from 40 -week-old LSL-Kras mice showed numerous tumors up to $3 \mathrm{~mm}$ in size. Age-matched LSL-Kras;LSL-HIF2 mice harbored significantly larger tumors, which occasionally reached $10 \mathrm{~mm}$ in diameter $(P<0.001$; Figure $1 \mathrm{~B}$ and Table 1$)$. These data show that expression of HIF $2 \alpha$ promotes the formation of larger tumors.

To determine whether the shortened survival of LSL-Kras;LSL-HIF2 mice resulted from an increase in lung tumor burden, we compared the total tumor area in LSL-Kras and LSL-Kras;LSL-HIF2 mice. To this end, we quantified low-power magnifications of a random section chosen from the right upper (apical) lobe (RUL) of mice for tumor area as well as total lung area. These measurements were used to determine the percentage of the RUL affected by tumor. At 40 weeks, LSL-Kras;LSL-HIF2 mice had a statistically significantly increased tumor burden relative to $L S L$-Kras mice $(P=0.031$; Figure $1 C)$. tumor burden found in the LSL-Kras;LSL-HIF2 mice is a reflection of alterations in tumor cell size, tumor edema or vascularity, or other increases in the non-neoplastic population of tumor-associated stromal cells (such as seen in Figure 2B). These data suggest that expression of HIF2dPA in Kras-induced lung carcinomas results in larger tumors and increased total lung tumor burden.

Kras-induced lung tumors expressing HIF2dPA have distinct gene expression changes. Numerous studies have used gene expression profiling to assess the transcriptional effects of VHL loss or HIF overexpression on cell lines $(17,19,41,42)$. We had previously examined the gene expression changes induced by $V b l$ loss or expression of stabilized forms of HIF $1 \alpha$ and HIF $2 \alpha$ within hepatocytes of mice (16). To validate that expression of stabilized HIF2 $\alpha$ resulted in significant changes in gene expression, we extracted total mRNA from 5 macrodissected tumors of both LSL-Kras and LSL-Kras;LSL-HIF2 mice and performed gene expression analysis using high-density oligonucleotide arrays. The gene expression patterns of LSL-Kras and LSL-Kras;LSL-HIF2 tumors were then examined by unsupervised hierarchical gene clustering. The transcriptional effects of HIF $2 \alpha$ were readily demonstrable by this approach; unsupervised analysis showed that the tumors clustered into two separate groups based on the presence or absence of HIF2dPA expression (Supplemental Figure 2) and were notable for the presence of several canonical HIF target genes such as Vegf, Tgfb1, Egln3, and Cxcl12 (Supplemental Figure 2). Therefore, our data indicate that expression of HIF2dPA within lung tumors results in activation of canonical HIF target genes and significant changes in gene expression.

HIF2 $\alpha$-producing lung tumors are poorly differentiated, with focal squamous differentiation, and display increased invasiveness. Histological examination of tumors from 40-week-old LSL-Kras mice, as expected, showed adenomatous alveolar hyperplasia and moderately to well-differentiated adenocarcinomas with bronchoalveolar carcinoma (BAC), acinar, and papillary features (Figure $2 \mathrm{~A})$, as described by others $(37,38,43)$. Lungs from age-matched LSL-Kras;LSL-HIF2 mice harbored two distinct subsets of tumors. Tumors of the first subset were reminiscent of those found in LSL-Kras mice. However, tumors of the second subset were poorly differentiated and displayed more cytologic atypia with pleomorphic histology (Figure 2A and Table 1) as well as increased fibrous stroma (Figure 2B and Table 1); occasional necrosis (Figure 2C); rare invasion of local structures (Table 1 ) such as the pleura (Figure 2D), myocardium (Figure 2E), and mediastinal adipose tissue (Figure 2F); or metastases to regional lymph nodes (Figure $2 \mathrm{G}$ ).

Poorly differentiated tumors were statistically significantly more frequent in LSL-Kras; LSL-HIF2 mice than their LSL-Kras counterparts $(P=0.027$; Table 1$)$. Examination of these tumors revealed occasional areas consistent with squamous differentiation. These 
A

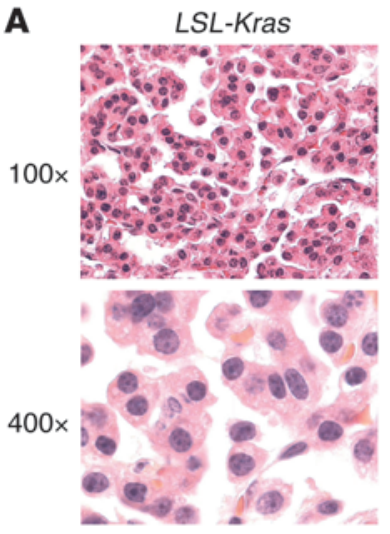

LSL-Kras;LSL-HIF2

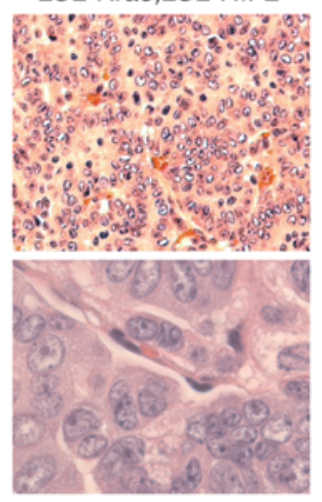

B
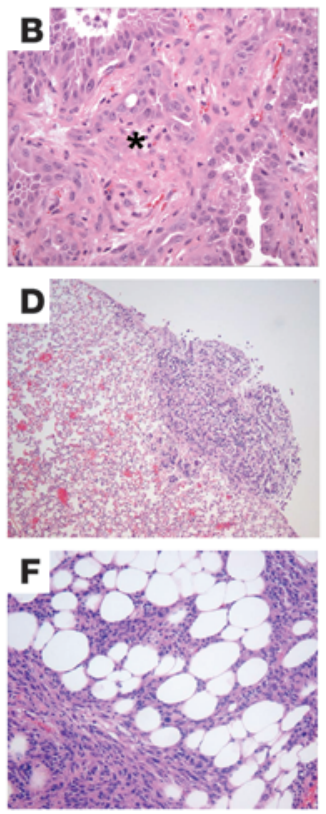
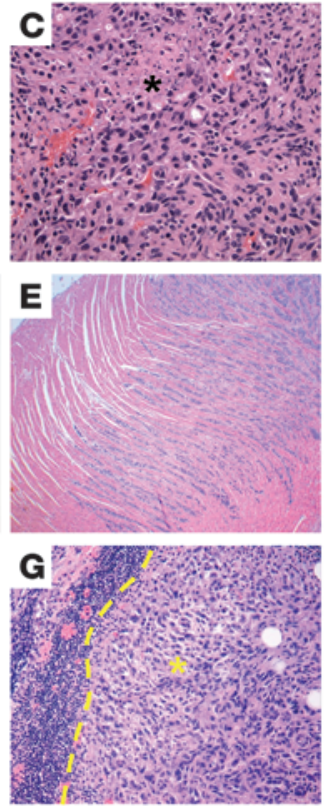

Figure 2

HIF2 $\alpha$-producing tumors are pleomorphic and invasive. (A) Representative low- and high-power photomicrographs of H\&E-stained lung tumors of 40-week-old LSL-Kras and LSL-Kras; LSL-HIF2 mice. (B-G) H\&E staining showing increased fibrous stroma (B, asterisk) as well as necrosis (C, asterisk) in a lung tumor from a 40-week-old LSL-Kras;LSL-HIF2 mouse. Tumors from 40-week-old LSL-Kras; LSL-HIF2 mice also showed features of invasiveness, such as invasion through the visceral pleura (D), myocardial invasion (E), direct invasion of mediastinal fat (F), and mediastinal lymph node metastases (G). Original magnification, $\times 100(\mathbf{B}, \mathbf{C}, \mathbf{F}$, and $\mathbf{G}) ; \times 40(\mathbf{D}$ and $\mathbf{E})$. The dashed yellow line indicates the division between normal lymph node and tumor; yellow asterisk indicates tumor.

cells had abundant eosinophilic cytoplasm, more centrally placed nuclei with prominent nucleoli, and less apparent polarity, features suggestive of squamous differentiation (Figure 3A). In addition, the areas that appeared morphologically squamous had high nuclear expression of the squamous cell marker p63 according to immunohistochemistry (Figure 3B), in a background of p63-negative adenocarcinoma cells (43).

The presence of squamous differentiation was interesting, as Kras-induced lung tumors are uniformly adenocarcinomas (37-40, 43). Even concurrent deletion of p53 or Pten, while promoting the development of higher-grade tumors, does not alter their histologic spectrum $(44,45)$. In contrast, we had previously described that inactivation of the $L k b 1$ tumor suppressor gene within Kras-induced lung tumors results in an expanded histologic spectrum including adeno-, squamous, and large cell carcinomas (43). We therefore queried the DNA microarray dataset generated on these Lkb1-deficient mouse lung tumors to see whether Hif2a gene expression correlated with histologic subtype. In keeping with the notion that HIF $2 \alpha$ promotes squamous differentiation, we found that Kras-induced, Lkb1deficient squamous cell or mixed adeno-squamous tumors had significantly higher levels of HIF $2 \alpha$ gene expression relative to Krasinduced, Lkb1-deficient adenocarcinomas (Supplemental Figure 3, $A$ and $B$ ). Therefore, the data indicate that HIF $2 \alpha$ gene expression is upregulated in mouse squamous cell lung carcinomas and that production of HIF $2 \alpha$ in Kras-induced lung tumors promotes squamous differentiation but not frank squamous cell carcinoma.

Kras-induced lung tumors expressing HIF2dPA have increased vascularity and blood flow. We next compared the vascularity of the autochthonous lung tumors by CD34 staining and quantification of microvessel density (MVD). Lungs from Ad-Cre-treated LSL-Kras and LSL-Kras;LSL-HIF2 mice were harvested, formalin fixed, and stained with antibodies against the endothelial cell marker CD34. The number of microvessels per high-power field was quantified by a reviewer blinded to the research protocol. As expected, the lung tumors from LSL-Kras;LSL-HIF2 mice displayed a significantly increased number of microvessels per high-power field relative to lung tumors from age-matched, littermate LSL-Kras control mice $(P<0.001$; Figure 4, A and B).

Increases in MVD do not necessarily translate into augmentation of blood flow, in part because of alterations in vascular permeability and heterogeneous blood flow within tumors (46). We and others have previously shown that the vessels formed by expression of stabilized forms of HIF in the skin are not conspicuously hyperpermeable $(16,47)$. However, these studies did not directly assess blood flow or the effects of HIF on tumor-associated blood vessels.

We asked whether the increase in vascular density seen by CD34 staining of HIF $2 \alpha$-producing lung tumors correlated with actual increases in blood flow. Because ultrasound waves are disrupted by pulmonary air, we were not able to image the primary tumors in situ in the lung, and therefore single-cell suspensions of primary tumors from LSL-Kras and LSL-Kras;LSL-HIF2 mice were injected subcutaneously into the flanks of nude mice. These tumors were then serially imaged with ultrasound and power Doppler sonography. Regions of blood flow were determined by power Doppler, while tumor volume was determined by $3 \mathrm{D}$ reconstruction of ultrasound images. The percent vascularity was determined as the ratio of blood flow to tumor volume. When matched for tumor volume, LSL-Kras;LSL-HIF2 tumors had a statistically significantly 
A

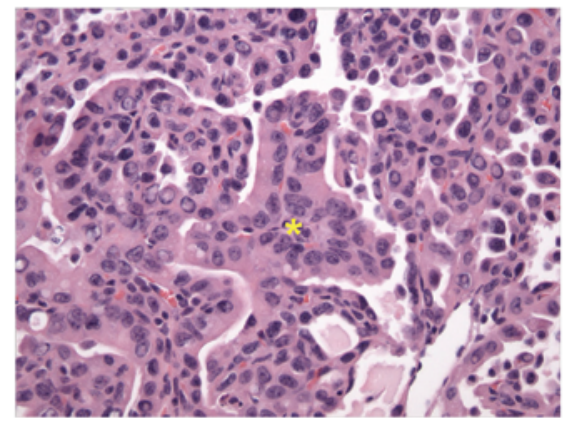

B

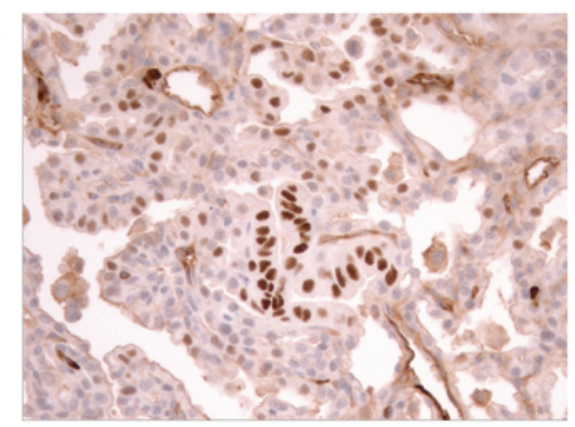

higher percent vascularity than LSL-Kras tumors $(P=0.028$; Figure $4, C$ and D). Additionally, while the percent vascularity of LSL-Kras tumors appeared to decline as the tumors enlarged (fitted exponential decay curve, $r^{2}=0.87$ ) the LSL-Kras; LSL-HIF2 tumors were more vascular at baseline, and this high percent vascularity persisted despite tumor growth (fitted exponential decay curve, $r^{2}=0.21$ ) (Figure 4E). Therefore, the increased MVD seen in tumors expressing stabilized HIF2 $\alpha$ appears to be paralleled by an increase in blood flow in tumor explants derived from these tumors.

Mice harboring HIF2 $\alpha$-producing lung tumors have elevated levels of CECs and CEPs. Increased numbers of CECs and CEPs have been detected in the peripheral blood of patients with solid tumors, including those with lung cancer (48-52). Several chemokines and cytokines known to be upregulated by HIF, such as VEGF and CXCL12, have been shown to be involved in the mobilization and homing of CECs and CEPs $(50,53-55)$. We therefore asked whether production of stabilized HIF2 $\alpha$ within lung tumors could stimulate the numbers of CECs and CEPs. To this end, we enumerated the numbers of CECs and CEPs by flow cytometry in the peripheral blood of LSL-Kras and LSL-Kras;LSL-HIF2 mice, 12 weeks after treatment with Ad-Cre. CECs were defined as CD45-CD117-CD $31^{+} \mathrm{VEGFR} 2^{+}$, while CEPs were defined as $\mathrm{CD} 45^{\mathrm{dim}} \mathrm{CD} 117^{+} \mathrm{CD} 31^{+} \mathrm{VEGFR} 2^{+}$. The lungs from these mice grossly appeared to have approximately the same tumor burden. Interestingly, the number of CECs and CEPs present in the peripheral blood of LSL-Kras;LSL-HIF2 mice was statistically significantly increased relative to that of LSL-Kras mice $(P=0.027$ and $P=0.014$, respectively) (Figure 5 , A and $\mathrm{B})$. This increase in CEPs, which are thought to be bone marrow derived, suggest that expression of stabilized HIF2 $\alpha$ within tumors promotes the mobilization of CEPs in an endocrine fashion.

HIF2 $\alpha$-producing tumors express markers of EMT in vivo. To better understand how HIF $2 \alpha$ activation within Kras-induced lung tumors might impart the phenotypes described, we performed gene set enrichment analysis (GSEA) using GSEA 2.0 (http:// www.broad.mit.edu/gsea) on our dataset of mouse lung tumors.

\section{Figure 3}

Squamous differentiation of HIF2 $\alpha$-producing lung tumors. (A) An H\&Estained lung tumor from a 40-week-old LSL-Kras; LSL-HIF2 mouse shows squamous features, including abundant eosinophilic cytoplasm, centrally placed nuclei with prominent nucleoli, and apparent loss of polarity (asterisk). (B) High-power photomicrograph of high nuclear p63 expression in a background of p63-negative adenocarcinoma as detected by immunohistochemistry of a LSL-Kras;LSL-HIF2 tumor. Original magnification, $\times 400$.

Specifically, an established collection of more than 100 cancerrelated, curated gene sets provided by the Molecular Signatures Database (MSigDB, broad.mit.edu/gsea/msigdb) as well as those collected from the literature (56-59) were used to interrogate the gene expression dataset in order to compare gene set enrichment in LSL-Kras and LSL-Kras;LSL-HIF2 tumors (Supplemental Table 2). We found 14 gene signatures to be enriched in HIF2 $\alpha$-producing tumors relative to their mutant Kras controls using a false discovery rate $(\mathrm{FDR})$ calculation $(P<0.05)$ to account for multiple hypothesis testing (Supplemental Figure 4). Notably, a significant number of the enriched gene sets were classifiers for either EMT or pathways known to induce EMT, such as the WNT/ $\beta$-catenin and TGF- $\beta$ pathways (60).

HIF has been implicated in promoting EMT $(1,61-64)$. Given our GSEA results and the invasive and metastatic nature of tumors derived from LSL-Kras;LSL-HIF2 mice, we hypothesized that they may express markers associated with EMT. We first asked whether these tumors had increased expression of genes implicated in inducing EMT by directly querying our gene expression dataset of LSL-Kras and LSL-Kras;LSL-HIF2 tumors. Interestingly, tumors expressing HIF2dPA had increased levels of several mRNA transcripts implicated causally or associated with EMT including Sip, Snail, Zeb1, and vimentin (all $P<0.01$ ) (Figure 6A). In addition, LSL-Kras;LSL-HIF2 tumors also stained positively for vimentin by immunohistochemistry (Figure 6B). These data are consistent with the notion that HIF $2 \alpha$ can induce a program of genes and markers involved in EMT in mouse lung carcinomas and suggest a possible reason why HIF2 $\alpha$-producing tumors are more invasive.

HIF2 $\alpha$ activation and EMT gene signatures are associated in human lung cancer. We asked whether the association between HIF2 $\alpha$ and EMT that we saw in our autochthonous murine lung tumors was present in human lung cancers. To that end, we first derived an HIF $2 \alpha$ gene signature from our mouse lung tumors. The gene expression profiles of the LSL-Kras and LSL-Kras;LSL-HIF2 tumors were compared using 2-class unpaired significance analysis of microarrays (SAM). The lowest FDR allowing the selection of 200 upregulated genes was chosen. Using an FDR of $0.71 \%$, we were able to define 396 significantly upregulated and 171 significantly downregulated genes. Human orthologs of the top 200 upregulated genes were determined and used to define an HIF $2 \alpha$ gene signature (Supplemental Table 1).

We next queried publicly available gene expression datasets of human lung cancer cell lines (65) or primary NSCLCs (66) for the presence or absence of our HIF2 $\alpha$ gene signature as well as the EMT, $\beta$-catenin, and TGF- $\beta$ gene signatures (67-69). To determine whether an association was present between HIF2 $\alpha$ activation and upregulation of gene signatures of EMT, $\beta$-catenin, and TGF- $\beta$, Genomica software (genomica.weizmann.ac.il) was used to calculate HIF $2 \alpha$, EMT, $\beta$-catenin, and TGF- $\beta$ enrichment scores for each tumor or cell line. The association between each of the 
A

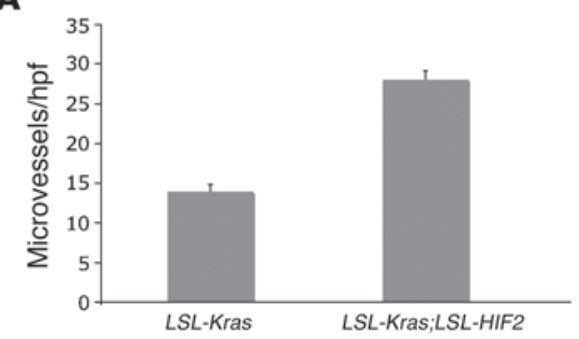

B

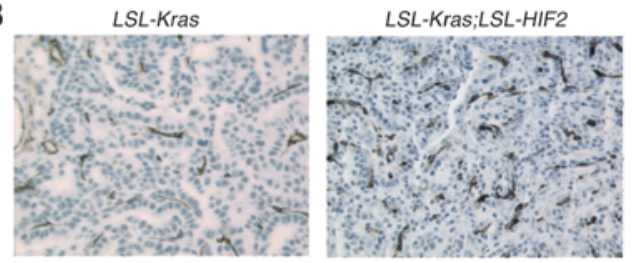

C

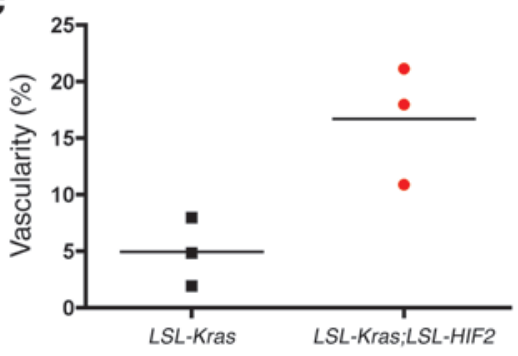

D
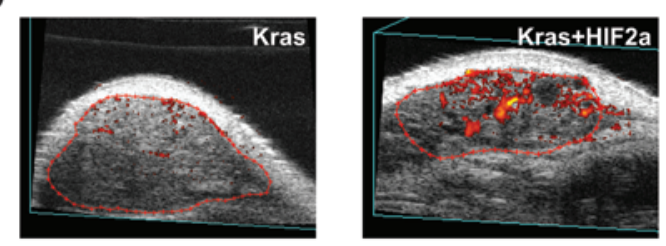

LSL-Kras;LSL-HIF2

$\mathbf{E}$

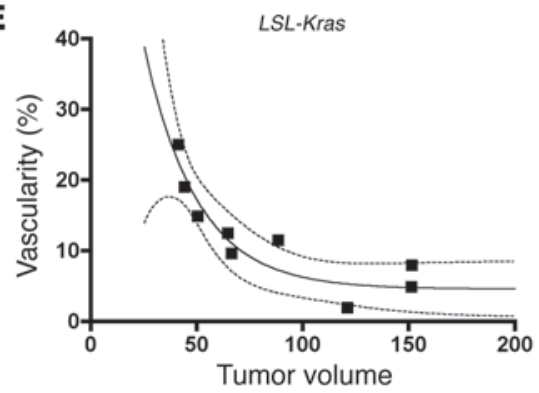

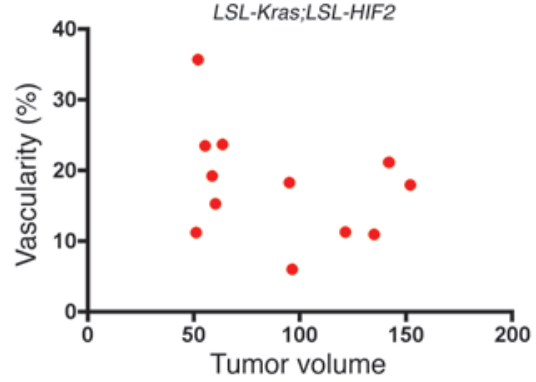

Figure 4

HIF2 $\alpha$-expressing tumors have increased vascularity and blood flow. (A) Quantification of microvessels per high-power field. Error bars indicate 1 SEM. $P<0.001$. (B) Immunohistochemistry for CD34 from lung tumors of $L S L-K r a s$ and LSL-Kras; $L S L-H I F 2$ mice. Original magnification, $\times 100$. (C) Quantification of percent vascularity of subcutaneously growing $L S L-K r a s$ and $L S L-K r a s ; L S L-H I F 2$ tumors. Lines indicate mean ( $P=0.028)$. (D and E) Representative pseudocolored Doppler ultrasound images of subcutaneously growing LSL-Kras and LSL-Kras;LSL-HIF2 tumors (D) and exponential decay curves of percent vascularity in relation to tumor volume (E). LSL-Kras: $r^{2}=0.87 ; L S L-K r a s ; L S L-H I F 2: r^{2}=0.21$.

gene signatures was then determined by calculating a Pearson correlation coefficient. Our HIF $2 \alpha$ gene signature correlated with enrichment of EMT, $\beta$-catenin, and TGF- $\beta$ gene signatures in both the lung cancer cell lines and the primary human NSCLCs (all $P<0.001$; Figure 7, A and B). Therefore, our HIF2 $\alpha$ gene signature appears to be applicable across species and is associated with EMT gene expression changes in human lung cancers.

\section{Discussion}

We found that production of stabilized HIF2 $\alpha$ in Kras-induced lung carcinomas resulted in larger, more invasive lung tumors and was associated with a shortened survival of mice harboring these tumors. Additionally, production of stabilized HIF2 $\alpha$ led to the development of tumors with a poorly differentiated and pleomorphic histology that expressed markers of EMT. Consistent with HIF's role in promoting angiogenesis, the HIF $2 \alpha$-producing lung tumors were found to have increased vascularity, and importantly, these tumors, when explanted into nude mice, were found to have increased blood flow at all stages of tumor development. Moreover, we detected higher levels of CECs and CEPs in the peripheral blood of mice harboring HIF $2 \alpha$-producing lung cancers, suggesting that tumors that produce HIF $2 \alpha$ can mobilize these cells from the tumor endothelium and bone marrow. Collectively, these results build upon the clinical data implicating HIF $2 \alpha$ as a negative prog- nostic factor in human NSCLC and demonstrate in an autochthonous cancer model that HIF $2 \alpha$ can promote tumor progression in cancers other than renal cell carcinoma.

The majority of studies reinforce the notion that HIF is a positive regulator of tumor growth. For example, cell lines having reduced HIF transcriptional activity secondary to knockout of Hifla or deficiency of Arnt grow more slowly under the skin of immunocompromised mice than wild-type controls (70-72). In genetically engineered mouse models, transgenic expression of Hif1a in basal keratinocytes facilitates the growth of HPV-16-induced cervical cancer, and conditional inactivation of Hifla in a mouse mammary tumor model restrains the growth of polyoma middle $\mathrm{T}$-induced breast tumors as well as pulmonary metastases $(73,74)$. However, other studies suggest that HIF may have a tumor suppressor role though induction of tumor cell apoptosis or cell-cycle arrest (75-78). Moreover, HIF can play starkly contrasting roles in tumor progression depending on the microenvironment in which the tumor cells are implanted, underscoring the importance of studying HIF in autochthonous tumor models (77). Our studies in the autochthonous lung cancers of genetically engineered mice support the notion that HIF $2 \alpha$ promotes solid tumor growth and aggressiveness.

In the setting of renal cell carcinoma, VHL loss and consequent upregulation of HIF is an early event (24). However, PVHL inactivation or HIF activation alone does not appear to be sufficient for 
A

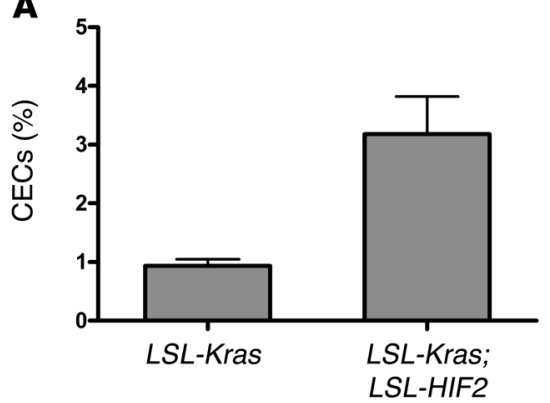

B

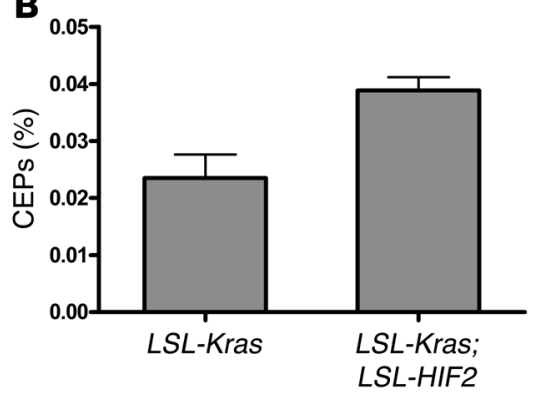

the formation of tumors in humans or mice (16, 24, 47, 79-82). Similarly, in our studies, we did not see tumor formation or significant histologic changes upon activation of only HIF $2 \alpha$ in the lungs of LSL-HIF2 mice (data not shown). Furthermore, the tumor multiplicity of lungs from $L S L$-Kras and LSL-Kras;LSL-HIF2 mice did not differ significantly, suggesting that even in the presence of an oncogenic mutation such as mutant Kras, HIF2 $\alpha$ does not initiate tumor formation.

In humans, non-small cell lung carcinomas can be subdivided into adeno-, squamous, and large cell carcinomas based on their histologic appearance. Kras-induced lung cancers in mice are uniformly well- to moderately differentiated adenocarcinomas. While concurrent loss of p53 or Pten appears to promote the development of higher-grade tumors, inactivation of these genes does not alter their histologic spectrum $(44,45)$. In contrast, $L k b 1$ inactivation within Kras-induced lung tumors results in an expanded histologic spectrum including adeno-, squamous, and large cell carcinomas (43). We noted that Lkb1-deficient squamous or mixed adenosquamous cell carcinomas expressed higher levels of Hif2a than Lkb1-deficient adenocarcinomas. We also noted that focal areas of HIF $2 \alpha$-producing tumors appeared to have squamous differentiation and stained positively for the squamous cell marker p63. Thus, while HIF $2 \alpha$ appears to modulate the histologic spectrum of lung cancers, the low penetrance of squamous differentiation seen in our studies suggests that HIF $2 \alpha$ expression alone is not sufficient to induce frank squamous cell carcinomas. These data also suggest that some of the phenotypic and transcriptional effects of $L k b 1$ loss may also be secondary to increased expression of HIF $2 \alpha$.

\section{Figure 6}

HIF2 $\alpha$-producing tumors display markers of EMT. (A) Fold change in gene expression of markers or inducers of EMT in LSL-Kras;LSL-HIF2 tumors relative to LSL-Kras tumors (Sip1, Snail, Zeb1, vimentin, and Twist). All $P<0.01$. Error bars indicate 1 SEM. (B) Expression of vimentin by immunohistochemistry in LSL-Kras and LSL-Kras; LSL-HIF2 tumors.

\section{Figure 5}

Mice harboring HIF2 $\alpha$-producing tumors have increased numbers of CECs and CEPs. Quantification of CECs (A) and CEPs (B) in the peripheral blood by flow cytometry expressed as a percentage of viable mononuclear cells. Error bars indicate 1 SEM. A: $P=0.027$; B: $P=0.014$.

The tumor microenvironment critically controls aspects of tumor growth and progression. In this regard, we present evidence that HIF $2 \alpha$ can increase tumor vascularity and may also augment tumor blood flow, suggesting that the neoangiogenesis induced by HIF $2 \alpha$-producing tumors results in a functional vasculature, as has been seen previously in non-neoplastic settings $(16,47)$. While the assessment of vascularity was performed on the autochthonous murine lung tumors, for technical reasons we had to assess the blood flow of HIF2 $\alpha$-producing lung tumors as tumor explants in which tumor cells are grown and examined under the skin of immunocompromised mice. Therefore, whether these studies adequately reflect the effects of HIF $2 \alpha$ within autochthonous lung tumors is uncertain.

While angiogenesis has been historically thought to be a local process, it has recently been appreciated that endothelium-derived endothelial cells (CECs) and their bone marrow-derived precursors (CEPs) can contribute to both physiologic and pathologic neoangiogenesis $(83,84)$. Interestingly, we detected increased numbers of both CECs and CEPs in the peripheral blood of mice harboring HIF2 $\alpha$-producing tumors relative to those expressing only mutant Kras. These results suggest that HIF $2 \alpha$ production within tumors is able to mobilize CECs and CEPs from the mature endothelium or bone marrow. Although it is tempting to speculate that this results from an increased elaboration of proangiogenic factors in HIF $2 \alpha-$ producing tumors, the precise mechanism whereby HIF $2 \alpha$ production is signaled to the bone marrow and the importance of these cells to tumor neovascularization remains to be determined.

Finally, the histologic and gene expression changes seen in the $H I F 2 d P A$-expressing tumors suggest that HIF $2 \alpha$ production on a backdrop of Kras activation results in more poorly differentiated tumors as well as induction of markers and mediators of EMT.

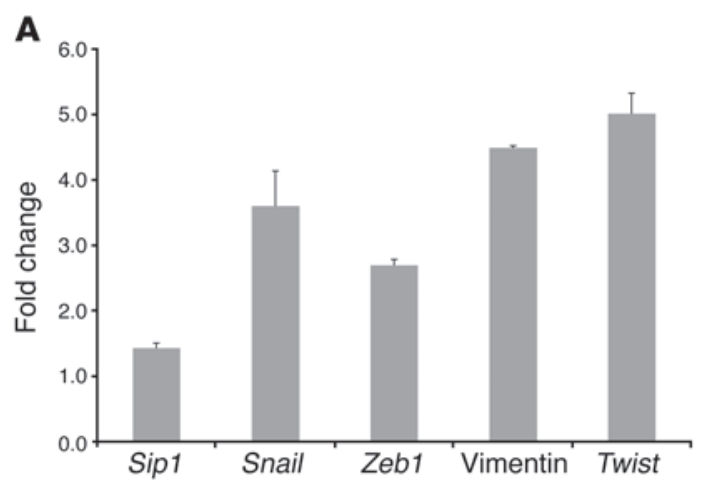

B
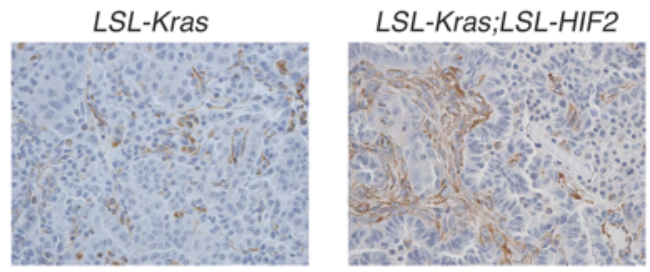
A Human lung cancer cell lines

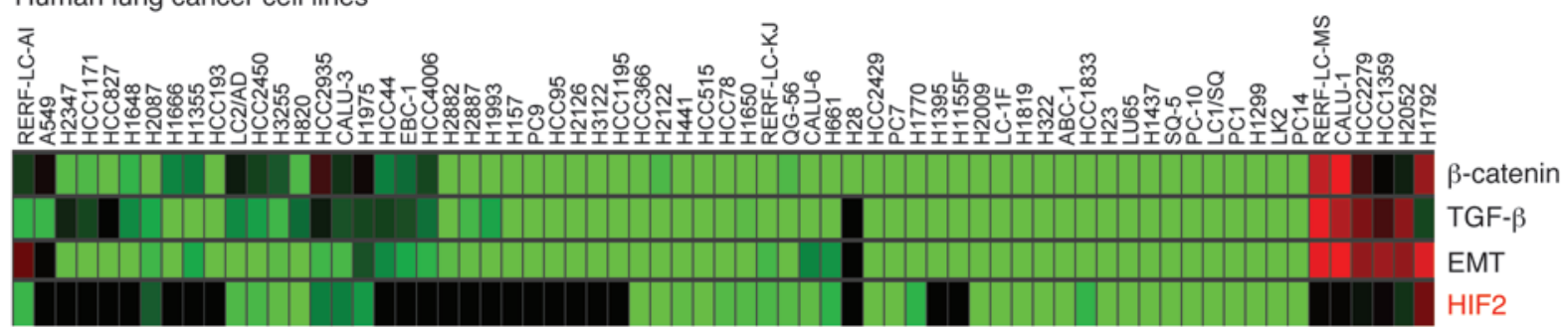

Primary human NSCLC

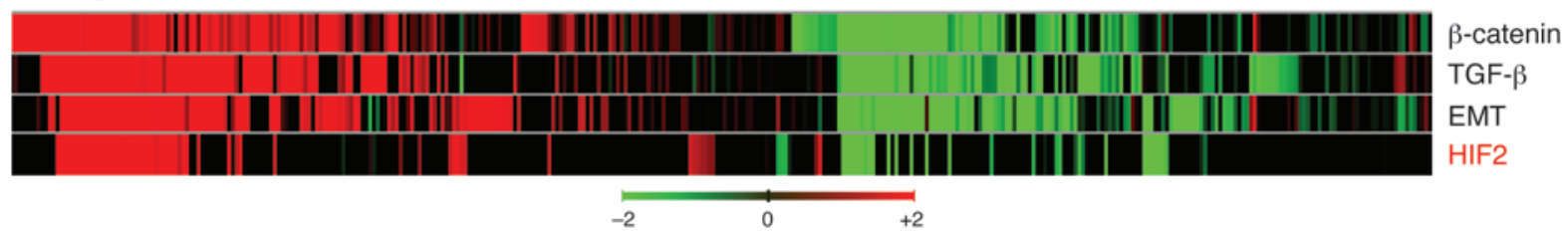

B
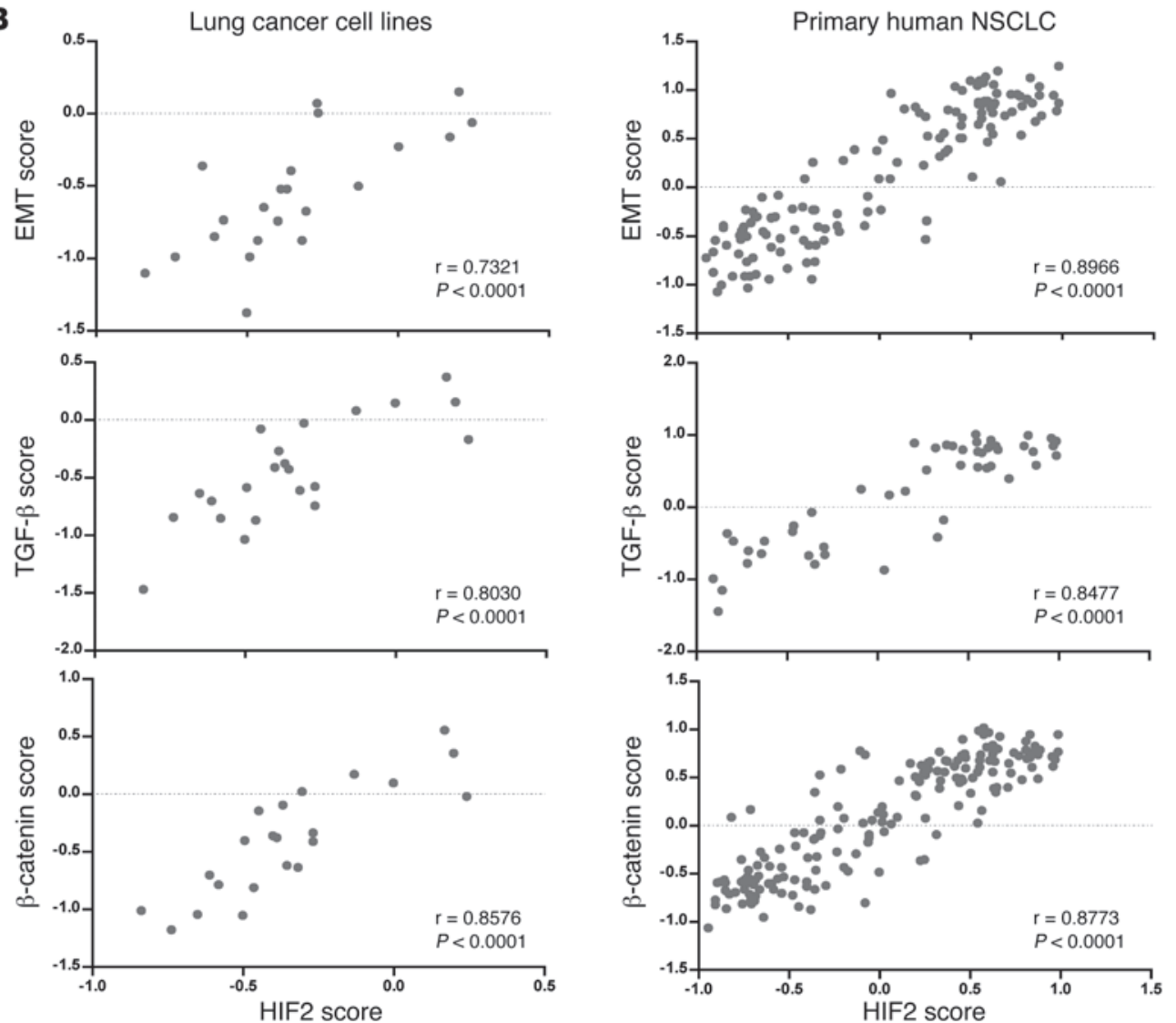

Figure 7

Association between HIF2 $\alpha$ activation and EMT gene signatures in human lung cancer. (A) Heat maps of relative enrichment scores of gene signatures of HIF2 $\alpha$, EMT, $\beta$-catenin, and TGF- $\beta$ in human lung cancer cell lines and primary human NSCLC. Tumors or cell lines are represented in columns. Gene signatures are represented in rows. Red indicates high enrichment score. Green indicates low enrichment score. (B) Graphical representation of correlation between EMT, $\beta$-catenin, and TGF- $\beta$ enrichment scores and HIF2 $\alpha$ enrichment score. Dots represent a single cell line or tumor.

These findings in autochthonous tumors extend a body of in vitro and xenograft data previously demonstrating that either HIF1 $\alpha$ or HIF $2 \alpha$ can induce EMT $(61-64,85)$. Moreover, cross-species analysis of our HIF $2 \alpha$ gene signature shows that HIF $2 \alpha$ activation correlates with gene signatures of EMT in human lung cancer, supporting the notion that HIF2 $\alpha$ initiates or is part of a genetic program resulting in EMT in human NSCLC.

HIF is predicted to transactivate a program of target genes that favor the progression of tumors. We have shown that HIF $2 \alpha$ activation results in the promotion of tumor growth but not tumor 
initiation and that HIF $2 \alpha$ 's effects on tumor size and invasiveness correlate with increased angiogenesis and expression of markers of EMT. Our data establish a causal link between HIF $2 \alpha$ and the shortened survival of patients, describe a mechanism whereby HIF2 $\alpha$ negatively impacts the overall survival of patients with NSCLC, and validate in autochthonous tumors that HIF $2 \alpha$ is a promoter of tumor growth.

\section{Methods}

Mouse colony maintenance and Ad-Cre administration. The LSL-Kras mice were provided by Tyler Jacks's laboratory (Massachusetts Institute of Technology, Cambridge, Massachusetts, USA), were maintained on a mixed background, and have been described thoroughly (38). The LSL-HIF2 mice were maintained on a C57BL/ 6 background and have been described previously (16). All mice were housed and treated in accordance with protocols approved by the institutional care and use committees for animal research at the University of North Carolina and Dana-Farber Cancer Institute. Ad-Cre was purchased from the University of Iowa and administered at a dose of $2.5 \times 10^{5}$ PFU intranasally as previously described (38).

Histological studies. Mice were sacrificed, and the left lungs were removed for studies requiring fresh tissue. The remaining right lung and mediastinal structures were inflated with neutral buffered $10 \%$ formalin (Sigma-Aldrich) and fixed overnight at $4{ }^{\circ} \mathrm{C}$. Fixed tissues were paraffin embedded and stained with H\&E by standard methods. For immunohistochemistry, 5 - $\mu \mathrm{m}$ sections were deparaffinized in xylene and rehydrated sequentially in ethanol. For antibodies requiring antigen retrieval, slides were boiled in $10 \mathrm{mM}$ sodium citrate ( $\mathrm{pH}$ 6.0) for 30 minutes. Sections were stained with antibodies generated against CD34 (rat, 1:50; Abcam), Ki67 (Vector Laboratories), TUNEL (S7100; Chemicon, Millipore), vimentin (BD Biosciences - Pharmingen), and p63 (Cell Signaling Technology). Quantification of microvessel density was performed using the Weidner method as previously described (86). Tumor area quantification was performed by ImageJ (http://rsbweb.nih. gov/ij/) measurements of tumor area and total lung area of 10 randomly chosen fields from the right apical lobe of each mouse. Tumor area was then expressed as percentage of total tumor area over total lung area. Tumor nodules were counted on one H\&E slide representing a complete cross section of the lungs per animal. Nodules that contained (a) fibrotic stroma generation, (b) necrosis, (c) morphologically high-grade tumor cells, and (d) tumor cells showing squamous morphology were also identified and counted on these same slides.

Ultrasound and Doppler studies. Tumor cell lines derived from lung tumors were implanted into the subcutaneous space of 8-week-old nude mice and serially imaged using ultrasound and Doppler (Vevo 770 microultrasound; VisualSonics). Tumor volume was determined in smaller tumors by $3 \mathrm{D}$ reconstruction of ultrasound images using the Vevo 770 software package. In larger tumors (exceeding the field of view of the probe), volume was determined by caliper measurement, using the formula vol $=0.5 \times \mathrm{L} \times \mathrm{W} \times \mathrm{W}$. Power Doppler mode imaging and percent vascularity was calculated using the Vevo 770 software package according to manufacturer's protocols.

Circulating endothelial and progenitor cell measurements. EDTA-anticoagulated whole blood was obtained from mice via cardiac puncture. Red blood cells were lysed with ACK lysis buffer $\left(0.15 \mathrm{M} \mathrm{NH}_{4} \mathrm{Cl}, 1 \mathrm{mM} \mathrm{KHCO} 3,0.1 \mathrm{mM}\right.$ EDTA, pH 7.3). The cells were then incubated with FcR Blocking antibody (catalog 553142; BD Biosciences - Pharmingen) according to the manufacturer's instructions, followed by incubation with the following fluorochrome-labeled monoclonal antibodies: anti-CD31 (PECAM-1)-FITC, anti-CD45-PE-Cy7, anti-CD117 (c-Kit)-APC, anti-VEGFR2 (Flk-1)-PE (all BD Biosciences - Pharmingen) or their appropriate isotype control antibodies. Dead cells were excluded by the use of 7-amino-actinomycin D (7AAD). CECs and CEPs, defined as CD $31^{+}$CD $45^{-}$CD $117-V E G F R 2^{+}$ and $\mathrm{CD} 31^{+} \mathrm{CD} 45^{-\mathrm{CD}} 117^{+} \mathrm{VEGFR} 2^{+}$, respectively, were quantified by flow cytometry (CyAn ADP flow cytometer; Beckman Coulter). All FACS data were analyzed by Flowjo software (Tree Star Inc.).

Microarray analysis. Mouse tumors were macrodissected, and total RNA was extracted. Labeled cDNA was synthesized using reverse transcription reactions carried out using $1 \mu \mathrm{g}$ of mRNA. Briefly, tumor mRNA was labeled with Cy5-dUTP, while the pooled cell line reference RNA (Stratagene) was labeled with Cy3-dUTP using the Agilent Technologies Low RNA Input Linear Amplification Kit and hybridized overnight at $65^{\circ} \mathrm{C}$ to an Agilent $4 \times 44 \mathrm{~K}$ complete mouse genome 60 mer oligonucleotide microarray. Arrays were washed and scanned using an Agilent Axon scanner and uploaded to the University of North Carolina microarray database, where the data are publicly available (https://genome.unc.edu/). Briefly, for analyses done using significance analysis of microarrays (SAM), data were excluded for genes with poor spot quality or with a mean intensity greater than twice the median background. Hierarchical clustering analyses were conducted using the program Cluster to do median-centered, average-linkage clustering, which was visualized using TreeView (http://jtreeview.sourceforge.net/). A total of 4,956 genes were selected from approximately 22,000 genes by filtering those with changes less than $0.5 \mathrm{SDs}$ from the median gene expression. All data shown in the heat map figures are median centered.

GSEA analysis of murine lung tumors. GSEA was performed using GSEA 2.0 downloaded from the Gene Set Enrichment Analysis website (http:// www.broad.mit.edu/gsea). The signal-to-noise metric and permutation of gene sets were used to rank the genes and calculate significance and FDR $(P>0.05, \mathrm{FDR}>0.05)$. Analysis was performed by collapsing probe sets to unique gene symbols and used to interrogate an established collection of curated gene sets provided by the Molecular Signatures Database (MSigDB, broad.mit.edu/gsea/msigdb) (87).

Gene signature analysis across buman lung cancer datasets. Overlapping genes among the HIF $2 \alpha$, EMT, WNT/ $\beta$-catenin, and TGF- $\beta$ activation gene signatures were first eliminated to remove bias during correlation comparisons. Full gene set lists are provided in Supplemental Table 1. Gene signature enrichment analysis and GSEA score assignment were performed as previously described (88) using Genomica software (genomica.weizmann. ac.il). Briefly, normalized microarray data from the mouse tumors, the human lung cancer cell line dataset (downloaded from the NCBI GEO website [http://www.ncbi.nlm.nih.gov/geo/], data sets GSE4824 and GDS1688), and the human primary lung NSCLC compendium (downloaded from the National Cancer Institute caArray website [caarraydb.nci. nih.gov], experiment ID 1015945236141280:1) (66) was first $\log _{2}$ transformed, and then the mean expression level for each gene across all samples in a given dataset was calculated. These mean values were subtracted from all data points to obtain a matrix where negative values represent below-mean expression and positive values the opposite. Each sample was first scored for genes whose expression was at least 2-fold above or below the mean expression level. We then assessed the fraction of over- or underexpressed genes that belong to each tested gene set, calculating a $P$ value for significant enrichment $(P<0.05)$. An FDR calculation was used to account for multiple hypothesis testing.

Pearson correlation. To quantify the association of the HIF $2 \alpha$ signature with the EMT, TGF- $\beta$, and WNT/ $\beta$-catenin signatures, we calculated the Pearson correlation between the scores across samples in the dataset. Scores were plotted in linear regression plots, and the probability of observing correlation was calculated by 1 -sided $t$ test. Pearson correlation and linear regression were calculated using GraphPad Prism 4.0 software. A $P$ value less than 0.05 was considered significant. 


\section{Acknowledgments}

We thank K. Rathmell and N. Bardeesy for useful discussions. This work was supported by the Cecily and Robert Harris Foundation (K.-K. Wong); the Joan Scarangello Foundation to Conquer Lung Cancer (K.-K. Wong and W.Y. Kim); the Flight Attendant Medical Research Institute (K.-K. Wong); the Melanoma Research Foundation (W.Y. Kim); the Department of Defense (W.Y. Kim); and NIH grants K08 AG024004 (to K.-K. Wong), R01 AG2400401 (to K.-K. Wong), R01 CA122794 (to K.-K. Wong), and K08 CA097203 (W.Y. Kim). We also acknowledge the assis- tance of the UNC Lineberger Genomics and Bioinformatics Core Facility.

Received for publication December 29, 2008, and accepted in revised form April 29, 2009.

Address correspondence to: William Y. Kim, M.D., Lineberger Comprehensive Cancer Center, University of North Carolina at Chapel Hill, 450 West Drive, CB\# 7295, Chapel Hill, North Carolina 27599, USA. Phone: (919) 966-4765; Fax: (919) 966-8212; E-mail: wykim@med.unc.edu.
1. Semenza, G.L. 2003. Targeting HIF-1 for cancer therapy. Nat. Rev. Cancer. 3:721-732.

2. Kaelin, W.G., Jr., and Ratcliffe, P.J. 2008. Oxygen sensing by metazoans: the central role of the HIF hydroxylase pathway. Mol. Cell. 30:393-402.

3. Hudson, C.C., et al. 2002. Regulation of hypoxiainducible factor 1alpha expression and function by the mammalian target of rapamycin. Mol. Cell. Biol. 22:7004-7014.

4. Thomas, G.V., et al. 2006. Hypoxia-inducible factor determines sensitivity to inhibitors of mTOR in kidney cancer. Nat. Med. 12:122-127.

5. Brugarolas, J.B., Vazquez, F., Reddy, A., Sellers, W.R., and Kaelin, W.G., Jr. 2003. TSC2 regulates VEGF through mTOR-dependent and -independent pathways. Cancer Cell. 4:147-158.

6. Zhong, H., et al. 2000. Modulation of hypoxiainducible factor 1alpha expression by the epidermal growth factor/phosphatidylinositol 3-kinase/ PTEN/AKT/FRAP pathway in human prostate cancer cells: implications for tumor angiogenesis and therapeutics. Cancer Res. 60:1541-1545.

7. Fukuda, R., Hirota, K., Fan, F., Jung, Y.D., Ellis, L.M., and Semenza, G.L. 2002. Insulin-like growth factor 1 induces hypoxia-inducible factor 1-mediated vascular endothelial growth factor expression, which is dependent on MAP kinase and phosphatidylinositol 3-kinase signaling in colon cancer cells. J. Biol. Chem. 277:38205-38211.

8. Fukuda, R., Kelly, B., and Semenza, G.L. 2003. Vascular endothelial growth factor gene expression in colon cancer cells exposed to prostaglandin E2 is mediated by hypoxia-inducible factor 1 . Cancer Res. 63:2330-2334.

9. Zundel, W., et al. 2000. Loss of PTEN facilitates HIF-1-mediated gene expression. Genes Dev. 14:391-396.

10. Brugarolas, J., et al. 2004. Regulation of mTOR function in response to hypoxia by REDD1 and the TSC1/TSC2 tumor suppressor complex. Genes Dev. 18:2893-2904.

11. Bertout, J.A., Patel, S.A., and Simon, M.C. 2008. The impact of $\mathrm{O} 2$ availability on human cancer. Nat. Rev. Cancer. 8:967-975.

12. Compernolle, V., et al. 2002. Loss of HIF-2alpha and inhibition of VEGF impair fetal lung maturation, whereas treatment with VEGF prevents fatal respiratory distress in premature mice. Nat. Med. 8:702-710.

13. Tian, H., Hammer, R.E., Matsumoto, A.M., Russell, D.W., and McKnight, S.L. 1998. The hypoxia-responsive transcription factor EPAS1 is essential for catecholamine homeostasis and protection against heart failure during embryonic development. Genes Dev. 12:3320-3324.

14. Iyer, N.V., et al. 1998. Cellular and developmental control of $\mathrm{O} 2$ homeostasis by hypoxia-inducible factor 1 alpha. Genes Dev. 12:149-162.

15. Gruber,M., etal. 2007. Acute postnatal ablation of Hif2alpha results in anemia. Proc. Natl. Acad. Sci. U. S. A. 104:2301-2306.

16. Kim, W.Y., et al. 2006. Failure to prolyl hydroxylate hypoxia-inducible factor alpha phenocopies VHL inactivation in vivo. EMBO J. 25:4650-4662.
17. Hu, C.J., Wang, L.Y., Chodosh, L.A., Keith, B., and Simon, M.C. 2003. Differential roles of hypoxiainducible factor 1alpha (HIF-1alpha) and HIF2alpha in hypoxic gene regulation. Mol. Cell. Biol. 23:9361-9374.

18. Hu, C.J., Sataur, A., Wang, L., Chen, H., and Simon, M.C. 2007. The N-terminal transactivation domain confers target gene specificity of hypoxia-inducible factors HIF-1alpha and HIF-2alpha. Mol. Biol. Cell. 18:4528-4542.

19. Raval, R.R., et al. 2005. Contrasting properties of hypoxia-inducible factor 1 (HIF-1) and HIF-2 in von Hippel-Lindau-associated renal cell carcinoma. Mol. Cell. Biol. 25:5675-5686.

20. Wang, V., Davis, D.A., Haque, M., Huang, L.E., and Yarchoan, R. 2005. Differential gene up-regulation by hypoxia-inducible factor-1alpha and hypoxiainducible factor-2alpha in HEK293T cells. Cancer Res. 65:3299-3306.

21. Kim, W.Y., and Kaelin, W.G. 2004. Role of VHL gene mutation in human cancer. J. Clin. Oncol. 22:4991-5004.

22. Maxwell, P.H., et al. 1999. The tumour suppressor protein VHL targets hypoxia-inducible factors for oxygen-dependent proteolysis. Nature. 399:271-275.

23. Gordan, J.D., et al. 2008. HIF-alpha effects on cMyc distinguish two subtypes of sporadic VHLdeficient clear cell renal carcinoma. Cancer Cell. 14:435-446.

24. Mandriota, K.T., et al. 2002. Hif activation identifies early lesion in vhl kidneys: evidence for sitespecific tumor suppressor function in the nephron. Cancer Cell. 1:459-468.

25. Kondo, K., Kim, W.Y., Lechpammer, M., and Kaelin, W.G., Jr. 2003. Inhibition of HIF2alpha is sufficient to suppress PVHL-defective tumor growth. PLoS Biol. 1:E83.

26. Kondo, K., Klco, J., Nakamura, E., Lechpammer, M., and Kaelin, W.G., Jr. 2002. Inhibition of HIF is necessary for tumor suppression by the von Hippel-Lindau protein. Cancer Cell. 1:237-246.

27. Zimmer, M., Doucette, D., Siddiqui, N., and Iliopoulos, O. 2004. Inhibition of hypoxia-inducible factor is sufficient for growth suppression of VHL-/- tumors. Mol. Cancer Res. 2:89-95.

28. Maranchie, J.K., et al. 2002. The contribution of VHL substrate binding and HIF1-alpha to the phenotype of VHL loss in renal cell carcinoma. Cancer Cell. 1:247-255.

29. Kaelin, W.G., Jr. 2008. The von Hippel-Lindau tumour suppressor protein: $\mathrm{O} 2$ sensing and cancer. Nat. Rev. Cancer. 8:865-873.

30. Swinson, D.E., et al. 2003. Carbonic anhydrase IX expression, a novel surrogate marker of tumor hypoxia, is associated with a poor prognosis in nonsmall-cell lung cancer. J. Clin. Oncol. 21:473-482.

31. Le, Q.T., et al. 2006. An evaluation of tumor oxygenation and gene expression in patients with early stage non-small cell lung cancers. Clin. Cancer Res. 12:1507-1514.

32. Lau, S.K., et al. 2007. Three-gene prognostic classifier for early-stage non small-cell lung cancer. J. Clin. Oncol. 25:5562-5569.

33. Giatromanolaki, A., et al. 2001. Relation of hypoxia inducible factor 1 alpha and 2 alpha in operable non-small cell lung cancer to angiogenic/molecular profile of tumours and survival. Br. J. Cancer. 85:881-890.

34. Kim, S.J., et al. 2005. Expression of HIF-1alpha, CA IX, VEGF, and MMP-9 in surgically resected nonsmall cell lung cancer. Lung Cancer. 49:325-335.

35. Socinski, M.A., et al. 2008. Multicenter, phase II trial of sunitinib in previously treated, advanced nonsmall-cell lung cancer. J. Clin. Oncol. 26:650-656.

36. Sandler, A., et al. 2006. Paclitaxel-carboplatin alone or with bevacizumab for non-small-cell lung cancer. N. Engl. J. Med. 355:2542-2550.

37. Meuwissen, R., Linn, S.C., van der Valk, M., Mooi, W.J., and Berns, A. 2001. Mouse model for lung tumorigenesis through Cre/lox controlled sporadic activation of the K-Ras oncogene. Oncogene. 20:6551-6558

38. Jackson, E.L., et al. 2001. Analysis of lung tumor initiation and progression using conditional expression of oncogenic K-ras. Genes Dev. 15:3243-3248.

39. Johnson, L., et al. 2001. Somatic activation of the $\mathrm{K}$-ras oncogene causes early onset lung cancer in mice. Nature. 410:1111-1116.

40. Fisher, G.H., et al. 2001. Induction and apoptotic regression of lung adenocarcinomas by regulation of a K-Ras transgene in the presence and absence of tumor suppressor genes. Genes Dev. 15:3249-3262.

41. Wykoff, C.C., et al. 2004. Gene array of VHL mutation and hypoxia shows novel hypoxia-induced genes and that cyclin D1 is a VHL target gene. $\mathrm{Br}$. J. Cancer. 90:1235-1243.

42. Wykoff, C.C., Pugh, C.W., Maxwell, P.H., Harris, A.L., and Ratcliffe, P.J. 2000. Identification of novel hypoxia dependent and independent target genes of the von Hippel-Lindau (VHL) tumour suppressor by mRNA differential expression profiling. Oncogene. 19:6297-6305.

43. Ji, H., et al. 2007. LKB1 modulates lung cancer differentiation and metastasis. Nature. 448:807-810.

44. Iwanaga, K., et al. 2008. Pten inactivation accelerates oncogenic K-ras-initiated tumorigenesis in a mouse model of lung cancer. Cancer Res. 68:1119-1127.

45. Jackson, E.L., et al. 2005. The differential effects of mutant p53 alleles on advanced murine lung cancer. Cancer Res. 65:10280-10288.

46. Jain, R.K., Munn, L.L., and Fukumura, D. 2002. Dissecting tumour pathophysiology using intravital microscopy. Nat. Rev. Cancer. 2:266-276.

47. Elson, D.A., et al. 2001. Induction of hypervascularity without leakage or inflammation in transgenic mice overexpressing hypoxia-inducible factor1alpha. Genes Dev. 15:2520-2532.

48. Dome, B., et al. 2006. Identification and clinical significance of circulating endothelial progenitor cells in human non-small cell lung cancer. Cancer Res. 66:7341-7347.

49. Ho, J.W., et al. 2006. Significance of circulating endothelial progenitor cells in hepatocellular carcinoma. Hepatology. 44:836-843.

50. Kollet, O., et al. 2001. Rapid and efficient homing of human CD34(+)CD38(-/low)CXCR4(+) stem and progenitor cells to the bone marrow and spleen of NOD/SCID and NOD/SCID/B2m(null) mice. 
Blood. 97:3283-3291.

51. Willett, C.G., et al. 2004. Direct evidence that the VEGF-specific antibody bevacizumab has antivascular effects in human rectal cancer. Nat. Med. 10:145-147.

52. Zheng, P.P., Hop, W.C., Luider, T.M., Sillevis Smitt, P.A., and Kros, J.M. 2007. Increased levels of circulating endothelial progenitor cells and circulating endothelial nitric oxide synthase in patients with gliomas. Ann. Neurol. 62:40-48.

53. De Falco, E., et al. 2004. SDF-1 involvement in endothelial phenotype and ischemia-induced recruitment of bone marrow progenitor cells. Blood. 104:3472-3482.

54. Asahara, T., et al. 1999. VEGF contributes to postnatal neovascularization by mobilizing bone marrow-derived endothelial progenitor cells. EMBO J. 18:3964-3972.

55. Furstenberger, G., et al. 2006. Circulating endothelial cells and angiogenic serum factors during neoadjuvant chemotherapy of primary breast cancer. Br.J. Cancer. 94:524-531.

56. Onder, T.T., et al. 2008. Loss of E-cadherin promotes metastasis via multiple downstream transcriptional pathways. Cancer Res. 68:3645-3654.

57. Ben-Porath, I., et al. 2008. An embryonic stem cell-like gene expression signature in poorly differentiated aggressive human tumors. Nat. Genet. 40:499-507.

58. Cole, M.F., Johnstone, S.E., Newman, J.J., Kagey, M.H., and Young, R.A. 2008. Tcf3 is an integral component of the core regulatory circuitry of embryonic stem cells. Genes Dev. 22:746-755.

59. Bild, A.H., et al. 2006. Oncogenic pathway signatures in human cancers as a guide to targeted therapies. Nature. 439:353-357.

60. Yang, J., and Weinberg, R.A. 2008. Epithelialmesenchymal transition: at the crossroads of development and tumor metastasis. Dev. Cell. 14:818-829.

61. Gort, E.H., et al. 2008. The TWIST1 oncogene is a direct target of hypoxia-inducible factor-2alpha. Oncogene. 27:1501-1510.

62. Yang, M.H., et al. 2008. Direct regulation of TWIST by HIF-1alpha promotes metastasis. Nat. Cell Biol. 10:295-305.

63. Esteban, M.A., et al. 2006. Regulation of E-cadherin expression by VHL and hypoxia-inducible factor. Cancer Res. 66:3567-3575.
64. Krishnamachary, B., et al. 2006. Hypoxia-inducible factor-1-dependent repression of E-cadherin in von Hippel-Lindau tumor suppressor-null renal cell carcinoma mediated by TCF3, ZFHX1A, and ZFHX1B. Cancer Res. 66:2725-2731.

65. Zhou, B.B., et al. 2006. Targeting ADAM-mediated ligand cleavage to inhibit HER3 and EGFR pathways in non-small cell lung cancer. Cancer Cell. 10:39-50.

66. Shedden, K., et al. 2008. Gene expression-based survival prediction in lung adenocarcinoma: a multi-site, blinded validation study. Nat. Med. 14:822-827.

67. Kenny, P.A., Enver, T., and Ashworth, A. 2005. Receptor and secreted targets of Wnt-1/beta-catenin signalling in mouse mammary epithelial cells. BMC Cancer. 5:3.

68. Verrecchia, F., Chu, M.L., and Mauviel, A. 2001. Identification of novel TGF-beta /Smad gene targets in dermal fibroblasts using a combined cDNA microarray/promoter transactivation approach. J. Biol. Chem. 276:17058-17062.

69. Jechlinger, M., et al. 2003. Expression profiling of epithelial plasticity in tumor progression. Oncogene. 22:7155-7169.

70. Ryan, H.E., et al. 2000. Hypoxia-inducible factor1 alpha is a positive factor in solid tumor growth. Cancer Res. 60:4010-4015.

71. Ryan, H.E., Lo, J., and Johnson, R.S. 1998. HIF-1 alpha is required for solid tumor formation and embryonic vascularization. EMBO J. 17:3005-3015.

72. Maxwell, P.H., et al. 1997. Hypoxia-inducible factor1 modulates gene expression in solid tumors and influences both angiogenesis and tumor growth. Proc. Natl. Acad. Sci. U. S. A. 94:8104-8109.

73. Liao, D., Corle, C., Seagroves, T.N., and Johnson, R.S. 2007. Hypoxia-inducible factor-1alpha is a key regulator of metastasis in a transgenic model of cancer initiation and progression. Cancer Res. 67:563-572.

74. Lu, Z.H., Wright, J.D., Belt, B., Cardiff, R.D., and Arbeit, J.M. 2007. Hypoxia-inducible factor-1 facilitates cervical cancer progression in human papillomavirus type 16 transgenic mice. Am. J. Pathol. 171:667-681.

75. Carmeliet, P., et al. 1998. Role of HIF-1alpha in hypoxia-mediated apoptosis, cell proliferation and tumour angiogenesis. Nature. 394:485-490.
76. Mack, F.A., et al. 2003. Loss of pVHL is sufficient to cause HIF dysregulation in primary cells but does not promote tumor growth. Cancer Cell. 3:75-88.

77. Blouw, B., et al. 2003. The hypoxic response of tumors is dependent on their microenvironment. Cancer Cell. 4:133-146.

78. Acker, T., et al. 2005. Genetic evidence for tumor suppressor role of HIF-2alpha. Cancer Cell. 8: $131-141$.

79. Haase, V.H., Glickman, J.N., Socolovsky, M., and Jaenisch, R. 2001. Vascular tumors in livers with targeted inactivation of the von Hippel-Lindau tumor suppressor. Proc. Natl. Acad. Sci. U. S. A. 98:1583-1588.

80. Ma, W., et al. 2003. Hepatic vascular tumors, angiectasis in multiple organs, and impaired spermatogenesis in mice with conditional inactivation of the VHL gene. Cancer Res. 63:5320-5328.

81. Rankin, E.B., Tomaszewski, J.E., and Haase, V.H. 2006. Renal cyst development in mice with conditional inactivation of the von Hippel-Lindau tumor suppressor. Cancer Res. 66:2576-2583.

82. Scortegagna, M., et al. 2008. HIF-1alpha regulates epithelial inflammation by cell autonomous NFkappaB activation and paracrine stromal remodeling. Blood. 111:3343-3354.

83. Bergers, G., and Benjamin, L.E. 2003. Tumorigenesis and the angiogenic switch. Nat. Rev. Cancer. 3:401-410.

84. Bertolini, F., Mancuso, P., and Kerbel, R.S. 2005. Circulating endothelial progenitor cells. N. Engl. J. Med. 353:2613-2616; author reply 2613-2616.

85. Evans, A.J., et al. 2007. VHL promotes E2 boxdependent E-cadherin transcription by HIF-mediated regulation of SIP1 and snail. Mol. Cell. Biol. 27:157-169.

86. Weidner, N., et al. 1992. Tumor angiogenesis: a new significant and independent prognostic indicator in early-stage breast carcinoma. J. Natl. Cancer Inst. 84:1875-1887.

87. Subramanian, A., et al. 2005. Gene set enrichment analysis: a knowledge-based approach for interpreting genome-wide expression profiles. Proc. Natl. Acad. Sci. U. S. A. 102:15545-15550.

88. Segal, E., Friedman, N., Kaminski, N., Regev, A., and Koller, D. 2005. From signatures to models: understanding cancer using microarrays. Nat. Genet. 37(Suppl.):S38-S45. 